\title{
Analysis of Adverse Reactions Related to Drugs and Vaccines Received at the National Centre for Pharmacovigilance from 2009 to 2016 in Togo
}

\author{
Yao Potchoo ${ }^{*}$, Mouhoudine Yerima1, Tante T. Gnandi ${ }^{1}$, Mounerou Salou${ }^{1}$, Aboudoulatif Diallo ${ }^{1}$, \\ Batoyema Bakoma ${ }^{1}$, Atani T. Nyansa ${ }^{2}$, Mireille Prince-David ${ }^{3}$ \\ ${ }^{1}$ Département des Sciences Pharmaceutiques, Faculté des Sciences de la Santé, Université de Lomé, Lomé, Togo \\ ${ }^{2}$ Direction de la Pharmacie, du Médicament et des Laboratoires, Ministère de la Santé et de la Protection Sociale, Lomé, Togo \\ ${ }^{3}$ Comité de Bioéthique et de la Recherche en Santé, Ministère de la Santé et de la Protection Sociale, Lomé, Togo \\ Email: *ypotchoo@hotmail.com
}

How to cite this paper: Potchoo, Y., Yerima, M., Gnandi, T.T., Salou, M., Diallo, A., Bakoma, B., Nyansa, A.T. and Prince-David, M. (2018) Analysis of Adverse Reactions Related to Drugs and Vaccines Received at the National Centre for Pharmacovigilance from 2009 to 2016 in Togo. Pharmacology \& Pharmacy, 9, 344-356.

https://doi.org/10.4236/pp.2018.98027

Received: June 30, 2018

Accepted: August 19, 2018

Published: August 22, 2018

Copyright $\odot 2018$ by authors and Scientific Research Publishing Inc. This work is licensed under the Creative Commons Attribution International License (CC BY 4.0).

http://creativecommons.org/licenses/by/4.0/

\section{(c) (i) Open Access}

\begin{abstract}
Objectives: To assess the received suspected adverse events occurring upon treatment with drugs and vaccines, at National Centre for Pharmacovigilance, in Togo, from 2009 to 2016. Methods: A crossover study was conducted in order to collect data about patients, drugs, suspected adverse events and notifiers. Suspected adverse events were classified using Med DRA 19.1. Notification's circumstances were classified into Public Health Programs' campaigns and routine practice. Data were collated into Excel spreadsheet and processed with SPSS software. Key Findings: Regional distribution is irregular. Of the 322 collected report forms, paramedics have notified $60.8 \%$ of the cases. Adult patients were the most represented (70.2\%). Public Health Programs campaigns provided $72.6 \%$ versus $27.4 \%$ for routine practice including Neglected Tropical Diseases (41.4\%), immunization (27.7\%), tuberculosis (25.9\%) and $4.5 \%$ for HIV. Skin disorders were the most prevalent suspected adverse events (147 sheets; $45.7 \%$ ) followed by general disorders and administration site disorders (29.8\%) and gastro-intestinal disorders (12.7\%). General anti-infective drugs for systemic use, antiparasites, and insecticides were the most reported class of medications (161 sheets; 44.7\%). Conclusions: A thorough follow-up of pharmacovigilance launched activities is needed to build a sustainable adverse effect's surveillance system and routine practice has to be strengthened.
\end{abstract}

\section{Keywords}

Medicines, Vaccines, Routine Health Care, Public Health Program, Adverse 
Events Reporting, Pharmacovigilance

\section{Introduction}

Pharmacovigilance (PV) is a science and activities relating to the detection, assessment, understanding and prevention of adverse effects (AE) or any other medicine-related problems. Its objectives are 1) preventing harm from adverse reactions in humans, arising from the use of authorized medicinal products within or outside the terms of marketing authorization or from occupational exposure and 2) promoting the safe and effective use of medicinal products, in particular through providing timely information about the safety of medicinal products to patients, healthcare professionals and the public [1].

Uppsala Monitoring Center (UMC), an independent and international research center affiliated to the World Health Organization (WHO), is the structure in charge of the monitoring medicines and promoting their adequate use since 1978 [2].

In sub-Saharan Africa, few studies covering a wide range of fields of the adverse events reporting process have been published. Ampuda et al., analyzing spontaneous reports in VigiBase ${ }^{\circledR}$ reported the following data: the main class of products in African Individual Case Safety Reports (ICSRs) includes the tree classes of reverse transcriptase inhibitors $(23.13 \%)$, antivirals for the treatment of HIV infections (5.50\%), combinations of sulfonamides and trimethoprim (2.98\%) and angiotensin-converting enzyme (ACE) inhibitors (2.42\%). The main product classes implicated in ICSRs from the rest of world are tumour necrosis factor-a (TNFa) inhibitors (5.29\%), topical nonsteroidal anti-inflammatory preparations (2.26\%), selective immunosuppressants (2.08\%), selective serotonin reuptake inhibitors $(2.04 \%)$ and 3-hydroxy-3-methylglutaryl-coenzyme (HMG $\mathrm{CoA}$ ) reductase inhibitors (1.85\%). The main SOCs reported from Africa versus the rest of world include skin and subcutaneous tissue disorders $(31.14 \%$ vs. $19.58 \%)$, general disorders and administration site conditions (20.91\% vs. $30.49 \%)$ and nervous system disorders (17.48\% vs. 19.13\%) [3].

In Togo, PV activities started in 2006. Information about AEs are gathered and recorded as cases on report forms (RFs), through reporters all over the country and are evaluated by a technical committee. Each case is assessed in order to establish a causal link between the observed AEs and the drugs' consumption. Once analyzed, cases are shared at a global level through VIGIFLOW ${ }^{\circledR}$, on one hand and with the reporters as feedback on the other hand, at the national level.

The PV system of Togo was, organized by the implementation of regulatory tools (creation decree), technical tools (RF, notification guide, assessment guide, supervision form, summary form) and human resources (national pharmacovigilance focal point, regional and district focal points, focal points in Hospitals) 
[4], from 2006 to 2011.

From January 2012 to May 2016, the National Centre for Pharmacovigilance (NCPV) did not organize any major activity due to lack of human resources [4].

The primary aim of this study, the first one in this topic in Togo, is to describe the collected notifications at the NCPV from 2009 to 2016. The secondary aims are to 1) assess the impact of follow up on the quality of the PV system, 2) describe the subgroups of data (patient, drug, $\mathrm{AE}$ and reporters) existing on the RFs, 3) identify the associated factors to AEs reporting in Togo.

\section{Methods}

\subsection{Study Design}

The received RFs at the NCPV, from 2009 to 2016, from Togo reporters, following Figure 1 circuit were included in our study. The RFs sent to the NCPV concerning cases occurring in other countries of the world have not been included.

\subsection{Data Collection and Analysis}

Eighteen (18) fields were collected from national adverse events reporting form for vaccines and other drugs. In comparison, adverse events reporting form for vaccines include 41 fields of which 12 are obligatory [5] and that for others medicines includes 33 fields [6]. The national reporting form required the following data:

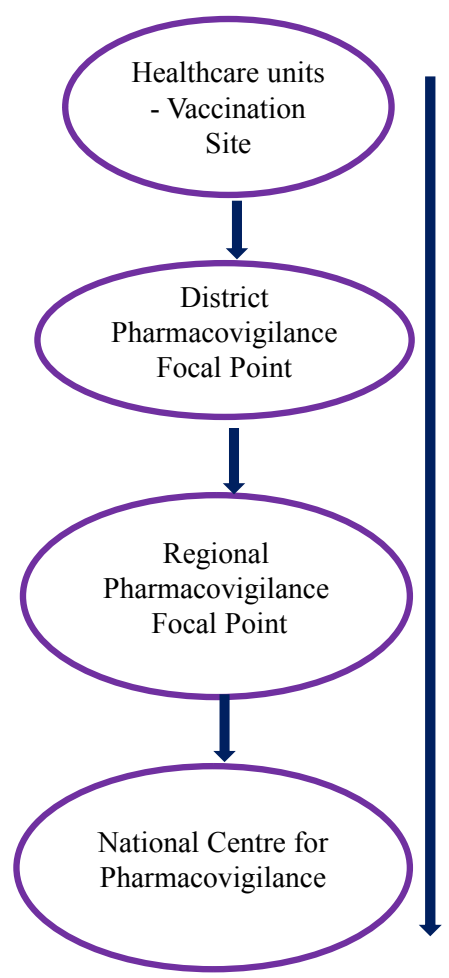

Figure 1. Adverse effect notification circuit in Togo. 
1) General data: year of notification;

2) Reporter data: profile, facility of practice, address/contact, regional area;

3) Patient data: age, sex, address, previous relevant medical conditions, laboratory investigations;

4) Product data: drug or vaccine, International Nonproprietary Name (INN), circumstances of product's use and;

5) Suspect adverse events (SAEs) data: description, onset time, outcome, received treatment, outcome in case of re-administration.

Year of notification was used to compare the RFs' numbers according to 2 periods (2009-2011 and 2012-2016), in order to determine whether the lack of integrated activities impacted the system.

The qualitative variables "region" and "reporter" were transformed into quantitative and binary variables.

Thus, the three regions of the north (Savannas, Kara, and Central) were named as "northern region" and those of the south (uplands, Maritime and Lome Commune) as "southern region".

Physicians and pharmacists have been grouped into medics and other healthcare professionals (nurses, health technicians, and midwifes) into paramedics.

Patients were divided into two age groups: children ( 0 to 15 years old) and adults (over 15 years old).

Vaccines have been considered as a distinct group from drugs, due to their exclusively preventive use. The identified INNs of drugs and vaccines were classified in accordance with the Anatomical Therapeutic and Chemical (ATC) nomenclature. The ATC nomenclature entails 14 main anatomical/pharmacological groups or first levels. Each major ATC group is divided into second levels that could be either pharmacological or therapeutic groups. The third and fourth levels are chemical, pharmacological or therapeutic subgroups and the fifth level is the chemical substance. Levels 2,3 and 4 are often used to identify pharmacological subgroups when it is considered more appropriate than therapeutic or chemical subgroups [7].

The collected SAEs were classified according to the severity criteria defined by WHO. A serious $\mathrm{AE}$ is defined as an $\mathrm{AE}$ which results in death, is life-threatening, requires in-patient hospitalization or prolongation of existing hospitalization, results in persistent or significant disability or incapacity, or is a congenital anomaly/birth defect [1]. No causal assessment was realized thus all the collected AEs were considered SAEs.

Notification circumstances were classified into two groups:

1) Routine practice (RP): they concern all the cases notified by healthcare professionals in either preventive or curative treatment for common conditions, for all kinds of population;

2) Public Health Programs (PHPs) mass treatment campaigns: they include notifications during campaigns organized by one or more PHPs (HIV, malaria, tuberculosis (TB), immunization), as a preventive measure, towards a specific target population. 
Suspect adverse events were coded according to the terminology of the Medical Dictionary for Regulatory Activities (MedDRA 19.1) [8]. It is an international medical terminology developed at the initiative of the International Conference on Harmonization ( $\mathrm{ICH}$ ), regarding the technical requirements for the registration of pharmaceuticals for human use. The codes refer to a group of MedDRA terms belonging to the primary system organ class (SOC). The SOC is a medical discipline classification.

Data was treated and analyzed with MICROSOFT ${ }^{\circ}$ Excel software 2013 SPSS software. The student test was used to compare the number of RF before and after 2012. The chi-2 test was used in the analysis of data and Fischer's exact test when the number was less than 5 . The significance threshold was set at $5 \%$.

\subsection{Ethical Considerations}

Patients and reporters' personal data were anonymous to preserve their confidentiality. The local Ethics Committee authorization was then, not required.

\section{Results}

\subsection{General Data}

A total of 322 RFs were collected from 2009 to 2016 (range 4 - 96, average 35.78 \pm 33.43 , median 27).

The completeness of the 18 fields on the 322 RFs is presented in Table 1 .

Table 1. Data completeness $(n=322)$.

\begin{tabular}{ccc}
\hline Data & $\mathrm{n}$ & $\%$ \\
\hline Patient age & 319 & 99.1 \\
Patient sex & 322 & 100.0 \\
Laboratory investigations Information & 1 & 0.3 \\
Patient address & 304 & 94.4 \\
Patient's previous medical relevant conditions & 44 & 13.7 \\
Patient contact & 164 & 50.9 \\
Product name & 322 & 100.0 \\
Product's use circumstances & 303 & 94.1 \\
Adverse Effect & 321 & 99.7 \\
Adverse Effect onset time & 298 & 92.6 \\
Adverse Effect outcome & 224 & 69.6 \\
Outcome in case of re-use & 12 & 3.7 \\
Product withdrawal information & 55 & 17.7 \\
Adverse Effect management & 161 & 50.0 \\
Reporter's profile & 290 & 90.1 \\
Reporter's contact & 318 & 98.8 \\
Reporter's region & 318 & 98.8 \\
Year of notification & 317 & 98.4 \\
\hline
\end{tabular}

$\mathrm{n}=$ Number of reports; $\%=$ Frequency. 
Eleven (11) fields out of 18 were filled out on more than $90 \%$ of report forms. Three (3) out of 18 fields were filled out in more than $50 \%$ of the RFs and the remaining 4 fields were filled out on less than $20 \%$ of the RFs.

The distribution of RFs received, per year is shown on Figure 2 with a significant decrease from 2012 to 2013, compared to the period before ( $p<0.05)$.

Notifiers' profiles are shown in Table 2. Paramedics reported two times (60.9\%) more than medics (29.2\%).

The notifier's profiles by regional area of practice are shown on Table 3 . The number of medics' RFs is 15 folds higher in the southern region than in the northern region of the country (56.5\% vs. $3.7 \%)$. As for paramedics', they reported two times more cases in the northern region than in the southern region ( $80.5 \%$ vs. $40.3 \%)$.

\subsection{Patient Data}

Males predominance was recorded, with 169 men out of $322(52.5 \%)$ and a male/female sex ratio of 1.1 .

Patients' ages ranged from 0.08 to 84 years old $(28.31 \pm 18.80$, median 27.0 years).

Table 4 shows the distribution of notifier profiles by patient age group. RFs for adult patients were higher than those for children $(70.2 \%$ versus $28.9 \%$; $p<$ $0.001)$

\subsection{Suspect Drugs of Adverse Events}

There were 243 RFs for medicines (74.5\%), and 79 (25.5\%) for vaccines. Eighty-three (83) RFs (25.8\%) were reported in routine practice while PHP campaigns resulted in 220 (74.2\%) notifications.

Several public health programs have been identified as shown in Table 5: neglected tropical diseases (41.4\%), immunization (27.7\%), tuberculosis (25.9\%) and HIV (4.5\%).

Table 6 shows the most prevalent reported products on the report forms. On

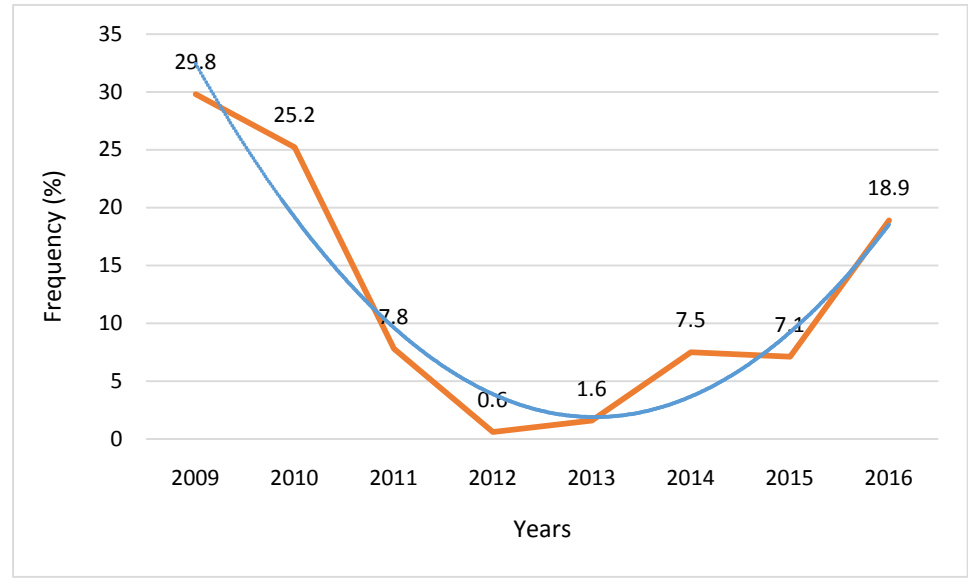

Figure 2. Report forms distribution per year $(\mathrm{n}=317)$. 
Table 2. Notifiers profile.

\begin{tabular}{ccc}
\hline Profile & Number & Frequency (\%) \\
\hline Medics & 94 & 29.2 \\
Paramedics & 196 & 60.9 \\
Unspecified & 32 & 9.9 \\
Total & 322 & 100.0 \\
\hline
\end{tabular}

Table 3. Notification distribution regarding to the notifier patterns and practice zone.

\begin{tabular}{|c|c|c|c|c|c|c|c|c|}
\hline \multirow{3}{*}{ Region } & \multicolumn{8}{|c|}{ Notifiers } \\
\hline & \multicolumn{2}{|c|}{ Medics } & \multicolumn{2}{|c|}{ Paramedics } & \multicolumn{2}{|c|}{ Unspecified } & \multicolumn{2}{|c|}{ Total } \\
\hline & $\mathrm{n}$ & $\%$ & $\mathrm{n}$ & $\%$ & $\mathrm{n}$ & $\%$ & $\mathrm{n}$ & $\%$ \\
\hline Northern & 6 & 3.7 & 132 & 80.5 & 26 & 15.9 & 164 & 50.9 \\
\hline Southern & 87 & 56.5 & 62 & 40.3 & 5 & 3.2 & 154 & 47.8 \\
\hline Unspecified & 1 & 25.0 & 2 & 50.0 & 1 & 25.0 & 4 & 1.2 \\
\hline Total & 94 & 29.2 & 196 & 60.9 & 32 & 9.9 & 322 & 100.0 \\
\hline
\end{tabular}

$\mathrm{n}=$ number; $\%=$ frequency.

Table 4. Notifiers distribution according to the notifiers' profiles and patient age group.

\begin{tabular}{ccccccccc}
\hline & \multicolumn{9}{c}{ Notifiers } \\
\cline { 2 - 8 } & \multicolumn{2}{c}{ Medics } & \multicolumn{2}{c}{ Paramedics } & \multicolumn{2}{c}{ Unspecified } & \multicolumn{2}{c}{ Total } \\
\cline { 2 - 9 } & $\mathrm{n}$ & $\%$ & $\mathrm{n}$ & $\%$ & $\mathrm{n}$ & $\%$ & $\mathrm{n}$ & $\%$ \\
\hline$[0 ; 15]$ & 3 & 3.2 & 73 & 78.5 & 17 & 18.3 & 93 & 28.9 \\
{$[15 ; 100]$} & 89 & 39.4 & 122 & 54.0 & 15 & 6.6 & 226 & 70.2 \\
Unspecified & 2 & 66.7 & 1 & 33.3 & 0 & 0.0 & 3 & 0.9 \\
Total & 94 & 29.2 & 196 & 60.9 & 32 & 9.9 & 322 & 100.0
\end{tabular}

$\mathrm{n}=$ headcount $\%$ = percentage.

Table 5. Report forms distributions by public health programs $(n=220)$.

\begin{tabular}{ccc}
\hline Public health programs & Number & Frequency (\%) \\
\hline Blindness & 1 & 0.5 \\
Neglected tropical diseases & 91 & 41.4 \\
Immunization & 61 & 27.7 \\
Tuberculosis & 57 & 25.9 \\
HIV & 10 & 4.5 \\
Total & 220 & 100.0 \\
\hline
\end{tabular}

the 322 collected report forms, 360 products were collected (1.1 product per report form). SAEs concerned mainly ivermectine (20.5\%), praziquantel (17.7\%), RHZE (15.8\%) and ACWY135 (15.5\%).

Table 7 presents the first level ATC group of the notified products. Anti-infectives for systemic use (44.7\%), pest control, insecticides $(28.9 \%)$ and cardiovascular system (11.9\%) were the main tree groups more frequently suspected. 
Table 6. Most common medicines reported on forms.

\begin{tabular}{ccc}
\hline INN & Headcount & Frequency (\%) \\
\hline Ivermectin & 66 & 20.5 \\
Praziquantel & 57 & 17.7 \\
RHZE & 51 & 15.8 \\
ACWY135 & 50 & 15.5 \\
Captopril & 13 & 4.0 \\
Amlodipine & 11 & 3.4 \\
D + T + C + P + HIB ${ }^{2}$ Vaccine & 11 & 3.4 \\
Inactive fragmented Influenza vaccine & 11 & 3.4 \\
Metformin & 9 & 2.8 \\
\hline
\end{tabular}

RHZE: Rifampicin + Isoniazid + Pyrazinamide + Ethambutol; ${ }^{2}$ VACCINE D + T + C + P + HIB: Pentavalent vaccine against diphtheria, tetanus, whooping cough, poliomyelitis and Haemophilus influenzae type b; INN: International nonapropriatery name.

Table 7. Distribution of drug groups according to the first level of ATC classification ( $\mathrm{n}=$ 360).

\begin{tabular}{ccc}
\hline ATC main group & Number & Frequency (\%) \\
\hline (J) Anti-infectives drugs for systemic use & 161 & 44.7 \\
(P) Pest Control, Insecticides & 104 & 28.9 \\
(C) Cardiovascular system & 43 & 11.9 \\
(A) Digestive tract and metabolism & 21 & 5.8 \\
(N) Nervous system & 16 & 4.4 \\
(B) Blood and hematopoietic organs & 8 & 2.2 \\
M) Muscle and skeleton & 3 & 0.8 \\
(R) Respiratory system & 3 & 0.8 \\
(H) Systemic Hormones, Excluded Sex Hormones & 1 & 0.3 \\
\hline
\end{tabular}

ATC: anatomical therapeutic and chemical.

\subsection{Suspect Adverse Effects Classification}

Table 8 shows the distribution of notifications, based on reporters and the seriousness of the SAE. Non serious SAEs represented $83.5 \%$ against serious SAEs (16.5\%). Medics notified $32.1 \%$ serious SAEs vs. $28.6 \%$ non-serious SAEs while paramedics notified $62.5 \%$ non-serious SAEs vs. $52.8 \%$ serious SAEs. There is no statistical link between the behavior of both medics and paramedics, in case of serious or non-serious SAEs $(\mathrm{p}=0.40)$.

Table 9 presents the SOC codes of suspect adverse event by frequency. By decreasing frequency reporting concerned skin and subcutaneous tissue disorders (45.7\%) followed by general disorders and administration site conditions (29.8\%), nervous system disorders (17.1\%), gastro-intestinal disorders $(17.1 \%)$ and musculoskeletal and systemic disorders $(7.8 \%)$. 
Table 8. Notifications distribution, according to the reporters and the seriousness of the adverse drug reaction.

\begin{tabular}{ccccccccc}
\hline & \multicolumn{8}{c}{ Notifiers } \\
\cline { 2 - 8 } Seriousness & \multicolumn{2}{c}{ Medics } & \multicolumn{2}{c}{ Paramedics } & Unspecified & Total \\
\cline { 2 - 8 } & $\mathrm{n}$ & $\%$ & $\mathrm{n}$ & $\%$ & $\mathrm{n}$ & $\%$ & $\mathrm{n}$ & $\%$ \\
\hline Serious & 17 & 32.1 & 28 & 52.8 & 8 & 15.1 & 53 & 16.5 \\
Non serious & 77 & 28.6 & 168 & 62.5 & 24 & 8.9 & 269 & 83.5 \\
Unspecified & 2 & 66.7 & 1 & 33.3 & 0 & 0.0 & 3 & 0.9 \\
Total & 94 & 29.2 & 196 & 60.9 & 32 & 9.9 & 322 & 100.0 \\
\hline
\end{tabular}

$\mathrm{n}=$ number $\%$ = percentage.

Table 9. Ten most common suspect adverse events according to the system organ class.

\begin{tabular}{ccc}
\hline System organ class & Number of sheet & Frequency (\%) \\
\hline Skin and subcutaneous tissue disorders & 147 & 45.7 \\
General disorders and administration site conditions & 96 & 29.8 \\
Nervous system disorders & 55 & 17.1 \\
Musculoskeletal and systemic disorders & 41 & 12.7 \\
Eye disorders & 25 & 7.8 \\
Renal and urinary disorders & 17 & 5.3 \\
Immune system disorders & 16 & 5.0 \\
Disorders of reproductive organs and breast & 13 & 4.0 \\
Respiratory, thoracic and mediastinal disorders & 11 & 3.4 \\
\hline
\end{tabular}

\section{Discussion}

\section{Study limits}

Pharmacovigilance activities were launched in 2007; our study was conducted from 2009 due to loss of the RFs before 2009.

The causal assessment was not realized because the available data were not sufficient for the assessment of all the cases.

The history of Public Health Programs' Campaigns was not available to allow us to assess whether the RFs distribution was proportional or not to treated population.

Pharmacovigilance activities were launched in 2007 in Togo with the advent of the strategies from World Health Organisation (WHO) to introduce PV through the PHPs in Africa (collaborations with malaria, HIV, TB, neglected diseases) [9].

Three hundred and twenty-two (322) reports were filled from 2009 to 2016 (range 4 -96, average 35.78, median 27). Compared with the number of spontaneous reports (556) received at Uganda's NCPV for the first 5 years from 2005 to 
2009 [10], our results confirm an under-reporting of our PV system that could be explained by the weakness of the system.

Concerning the completeness of the filled fields, eleven (11) out of 18 fields were filled on more than $90 \%$ of the report forms. Three (3) out of 18 fields were filled in more than $50 \%$ of the RFs and notifications and the remaining 4 fields were less than $20 \%$ of them. This high rate of completeness of forms is explained by the validation of report forms established by the NCPV team. The pharmacovigilance focal points were trained to validate the report forms and to transmit only those which had a sufficient level of data, to make the record usable [4]. The significant drop down of the number of RFs from 2012 to 2013 can be explained by the lack of human resources at the NCPV during the period from 2009 to 2016 [4].

Medics' reports from the southern part of the country are 15 folds that from the northern part whereas paramedics' reports from the northern region are two folds that from the southern. This can be explained by the concentration of medics in the South of the country and paramedics in the North.

A predominance of males is recorded in our study with $52.5 \%$ while identical proportions of females (57\% Africa and the rest of world) and males (37\% Africa and the rest of world) were reported from VigiBase ${ }^{\circledR}$ [3]. That difference related to our result could be explain by the fact of hazard. Our results show that report forms in adult patients represented two third against one third in children. Carbonin et al. [11] and Bégaud et al. [12] also have noticed that the notification rate increased with age. This result confirm that of Ampadu et al., who reported that the 18 - 44 years age group dominated individual case safety reports (ICSRs) from Africa, while the 45 - 64 years age group dominated the rest of the world [3].

One third of report forms were carried out during routine practice while PHP campaigns resulted in two third notifications. A possible explanation may be the rigorous monitoring of activities implemented by PHP during their campaigns.

There is no statistical link between the behavior of both medics and paramedics, in case of serious or non-serious SAEs.

General anti-infectives drugs for systemic use, antiparasitic agents and insecticides, cardiovascular system and digestive tract and metabolism were the pharmacological groups of the most reported drugs in our study. The most frequently mentioned medication classes associated with ADRs in the past 4 weeks in Uganda were antibiotics (38\%), antiretroviral agents (23\%), antimalarials (15\%, 15 of which implicated quinine), analgesics (9\%) and others (15\%) [10]. The main class of products in African ICSRs are nucleoside and nucleotide reverse transcriptase inhibitors (14.04\%), nonnucleoside reverse transcriptase inhibitors $(9.09 \%)$, antivirals for the treatment of HIV infections (5.50\%), combinations of sulfonamides and trimethoprim $(2.98 \%)$ and angiotensin-converting enzyme (ACE) inhibitors (2.42\%). Compared to the rest of the world, the main product classes implicated in ICSRs are tumour necrosis factor-a (TNFa) inhi- 
bitors (5.29\%), topical non-steroidal anti-inflammatory preparations $(2.26 \%)$, selective immunosuppressants $(2.08 \%)$, selective serotonin reuptake inhibitors (2.04\%) and HMG CoA reductase inhibitors (1.85\%) [3]. Our study results are consistent with the epidemiological context of the country. The SAEs reported (skin and sub cutaneous tissue disorders, general disorders and administration site conditions, nervous system disorders, gastro-intestinal disorders, musculoskeletal and systemic disorders...) can be related to main adverse effects reported in literature for anti-infectives drugs for systemic use such as: ivermectine (pruritus, edema, arthralgia, headache, popular eruptions) [13]; praziquantel (nausea and abdominal pain or discomfort, headache, giddiness or drowsiness) [14]; RHZE (gastric intolerance, skin changes, join pain) [15] and vaccine ACWY 135 (headache, fatigue, soreness, erythema) [16]. Furthermore, in a study among inpatients, Fofana et al. [17], reported similar SOCs excluding metabolic disorders such as skin (47.33\%), metabolic (22.14\%), neuromuscular $(10.68 \%)$ and digestive $(8.4 \%)$. The drugs implicated in skin adverse reactions in this study (causal link established) were sulfonylurea (47.37\%), penicillin (13.16\%), non-steroidal anti-inflammatory agents (13.16\%) or tramadol (7.89\%). The difference observed in the two study could be due at least to the sitting (national vs hospital) and the eligible population (all individual vs inpatients). Related to international data, the main SOCs reported from Africa versus the rest of world include skin and subcutaneous tissue disorders (31.14\% vs. 19.58\%), general disorders and administration site conditions (20.91\% vs. $30.49 \%)$ and nervous system disorders (17.48\% vs. $19.13 \%$ ) [3]. The main SOCs profile of our findings is similar to that reported from Africa.

\section{Conclusions}

In conclusion, this cross-sectional study conducted on SAEs' notifications in Togo, pointed out that paramedics are more involved in PV activities than medics. Public health program campaigns had led to more notifications than routine practice. Regional notifications distribution is neither equal nor proportional to the population showing heterogeneous practices among professionals. The lack of human resources from 2012 to 2016 created a significant dropdown in the notification rate.

The most frequently affected SOC was the skin disorders and the subcutaneous tissue were the most frequently reported.

Among the ATC classes of the drugs with the highest number of RFs, the most important are the general anti-infectives drugs for systemic use and antiparasitics and insecticides including the implication of the Ivermectin, Praziquantel, the association RHZE and ACYW135 vaccine.

Overall, the analysis of the results revealed some important determining factors. Statistically significant factors were the area of exercise, the age of the patient, health product consumption circumstance and the type of health products. These determining factors could lead to some interventions to strengthen the national pharmacovigilance system. 


\section{Conflicts of Interest}

The authors declare no conflicts of interest regarding the publication of this paper.

\section{References}

[1] European Medicines Agency (2017) Guideline on Good Pharmacovigilance Practices (GVP). Annex I-Definitions (Rev 4). EMA/876333/2011 Rev 4, 22.

http://www.ema.europa.eu/docs/en_GB/document_library/Scientific_guideline/201 3/05/WC500143294.pdf

[2] Pajot, F. (2005) Objectifs et méthodes du suivi post-commercialisation d'un médicament. Thèse, Université de Nantes, Faculté de pharmacie, Nantes, 101 p.

[3] Ampadu, H., Hoekman, J., de Bruin, M.L., Pal, S.N., Olsson, S., Sartori, D., Leufkens, H.G.M. and Dodoo, A.N.O. (2016) Adverse Drug Reaction Reporting in Africa and a Comparison of Individual Case Safety Report Characteristics between Africa and the Rest of the World: Analyses of Spontaneous Reports in VigiBase. Drug Safety, 39, 335-345. https://doi.org/10.1007/s40264-015-0387-4

[4] Centre National de Pharmacovigilance Togo (2011) Trimester Activities Report 4.

[5] WHO (2018) Formulaire de notification des manifestations postvaccinales indésirables (MAPI).

http://www.who.int/vaccine_safety/initiative/tools/AEFI_reporting_form_FR_Jan20 16.pdf?ua=1

[6] WHO (2012) Assurer la sécurité des interventions de chimioprévention pour la lutte contre les maladies tropicales négligées: Conseils pratiques à l'intention des administrateurs de programmes nationaux sur la prévention, le dépistage, et la prise en charge des événements indésirables graves. 58.

http://apps.who.int/iris/bitstream/handle/10665/75185/9789242502190_fre.pdf;jsess ionid=20B5EEA5A262BED2BBB12288E8011BB8? sequence $=1$

[7] WHO Collaborating Centre for Drug Statistics Methodology Norwegian Institute of Public Health (2018) ATC/DDD Principles.

https://www.whocc.no/atc/structure_and_principles/

[8] World Health Organization (2018) Essential Medicine and Products. http://www.who.int/medicines/areas/quality_safety/safety_efficacy/pharmvigi/en/

[9] Bencheikh, R.S., Ouledghriss, R., Skalli, S. and Alj, L. (2018) Pharmacovigilance Systems in Africa North Africa/Sub-Saharan Africa.

https://www.dndi.org/wp-content/uploads/2009/11/r_soulaymani_pharmacovigilan ce-Africa_astmh2009.pdf

[10] Kiguba, R., Karamagi, C., Waako, P., Ndagije, H.B. and Bird, S.M. (2014) Recognition and Reporting of Suspected Adverse Drug Reactions by Surveyed Healthcare Professionals in Uganda: Key Determinants. BMJ Open, 4, e005869.

https://doi.org/10.1136/bmjopen-2014-005869

[11] Carbonin, P., Bernabei, R. and Sgadari, A. (1991) Is Age an Independent Risk Factor of Adverse Drug Reactions in Hospitalized Medical Patients? Journal of the American Geriatrics Society, 39, 1093-1099. https://doi.org/10.1111/j.1532-5415.1991.tb02875.x

[12] Bégaud, B., Martin, K., Fourrier, A. and Haramburu, F. (2002) Does Age Increase the Risk of Adverse Drug Reactions? British Journal of Clinical Pharmacology, 54, 550-552. https://doi.org/10.1046/j.1365-2125.2002.01694.x

[13] Prod'hom, J., Boussinesq, M., Fobi, G., Prud'hom, J.M., Enyong, P., Lafleur, C. and Quillévéré, D. (1991) Lutte contre l’onchocercose par ivermectine: Résultats d'une 
campagne de masse au Nord-Cameroun. Bulletin de I Organisation Mondiale de la Santé, 69, 443-450.

[14] Katz, N., Rocha, R.S. and Chaves, A. (1979) Preliminary Trials with Praziquantel in Human Infections Due to Schistosoma mansoni. Bulletin de l'Organisation Mondiale de la Santé, 57, 781-785.

[15] Rabahi, M.F., Rodrigues da Silva, J.J.L., Galvão Ferreira, A.C., Schwartz Tanus-Silva, D.G. and Conde, M.B. (2017) Tuberculosis Treatment. Jornal Brasileiro de Pneumologia, 43, 472-486. https://doi.org/10.1590/s1806-37562016000000388

[16] Ambrosch, F., Wiedermann, G., Crooy, P. and George, A.M. (1983) Immunogenicity and Side Effects of a New Tetravalent Meningococcal Polysaccharide Vaccine. Bulletin de P Organisation Mondiale de la Santé, 61, 317-323.

[17] Fofana, S., Yaméogo, T.M., Zongo, R.F.E., Kyelem, C.G., Ouédraogo, R.W.-L., Diallo, B., Kaboré, L., Sombie, I., Ilboudo, A., Ouédraogo, M., Sanou, B. and Guissou, I.P. (2017) Pharmacovigilance at University Teaching Hospital Souro Sanou: Study of Spontaneous Reports of Adverse Drug Reactions. World Journal of Pharmaceutical Research, 6, 202-215. 